\title{
Multiple-site bleeding at pleural adhesions and massive hemothorax following percutaneous coronary intervention with stent implantation: A case report
}

\author{
XIAOLI LUO* ${ }^{*}$ WEIBIN SHI* ${ }^{*}$ XIAOQUN ZHANG, XIAOLI YANG, \\ WEI WANG, CHUNYU ZENG and HONGYONG WANG \\ Department of Cardiovascular Medicine, Institute of Field Surgery, \\ Daping Hospital of The Third Military Medical University, Chongqing 400042, P.R. China
}

Received April 5, 2016; Accepted March 6, 2017

DOI: $10.3892 /$ etm.2018.5685

\begin{abstract}
An elderly male patient with coronary heart disease underwent coronary angiography, which revealed bilateral severe coronary artery stenosis. Four stents were implanted, and at $5 \mathrm{~h}$ post-surgery, typical hemorrhagic shock appeared. Echocardiography verified heavy bleeding in the right thoracic cavity. Emergency angiography excluded injury or perforation of the coronary artery, aorta, right subclavian artery and brachiocephalic artery. Considering the patient's history of chronic obstructive pulmonary disease and severe cough during the operation, it was suspected that hemothorax was induced by a blood vessel rupture in the pleura. Video-assisted thoracic examination confirmed the tearing of pleural adhesion bands and bleeding at three sites in the pulmonary pleura and parietal pleura. According to the reported case, bleeding as a result of the laceration of pleural adhesions is an important cause of hemothorax that should be considered after exclusion of other common causes of pleural hemorrhage.
\end{abstract}

\section{Introduction}

Spontaneous hemopneumothorax refers to an accumulation of $>400 \mathrm{ml}$ blood in the pleural cavity without obvious trauma or other causes. Spontaneous hemopneumothorax is a rare, potentially life-threatening condition occurring predominantly in adolescents and adults, with a prevalence ratio of $0.0025 \%$, or $1-12 \%$ of all spontaneous pneumothoraces (1). Bleeding

Correspondence to: Dr Hongyong Wang, Department of Cardiovascular Medicine, Institute of Field Surgery, Daping Hospital of The Third Military Medical University, 10 Changjiang Branch Road, Chongqing 400042, P.R. China

E-mail: cqwhysir@sina.com

*Contributed equally

Key words: bleeding, pleural adhesion, hemothorax, percutaneous coronary intervention, surgery at the pleural adhesions is a major reason for spontaneous hemopneumothorax (2-4), which can lead to mortality due to acute respiratory and circulatory dysfunction if the condition becomes aggravated. This condition is a rare thoracic emergency in the clinical practice, and thus has a high rate of missed diagnosis (5). Non-surgical therapy of bleeding at the adhesion bands of the pleura is not effective and results in relapse; thus, active surgical treatment is the best therapy available $(6,7)$.

Percutaneous coronary intervention (PCI) for coronary revascularization has been an extensively used medical therapy for chronic and acute coronary artery disease (8). More than 50,000 PCI procedures are performed annually in China (9). Spontaneous hemopneumothorax is not listed as one of the complications observed during PCI. Probably due to the low incidence of spontaneous hemopneumothorax, the occurrence of spontaneous hemopneumothorax during the PCI therapy is rarely reported (9).

The present study reports a case of bleeding at multiple pleural adhesion bands following percutaneous coronary intervention, causing severe hemothorax. The reason behind hemothorax occurrence in such cases can be easily neglected, thus the present study suggests that this condition should be carefully considered by the clinicians in order to avoid misdiagnosis. The study was approval by the Ethics Committee of the Institute of Field Surgery, Daping Hospital of the Third Military Medical University (Chongqing, China). Written informed consent was obtained from the patient prior to inclusion in the current study.

\section{Case report}

A 76-year-old male patient was referred to Daping Hospital due to 3 years of exertional chest distress, accompanied by chest pain and slight cough that persisted for 1 week. The patient complained of recurring precordial chest pain resulting from exhaustion 3 years earlier, which lasted several minutes each time, but was relieved after rest. The patient did not seek treatment at that time. Precordial chest pain was not accompanied by headache, dizziness, cough, sputum, abdominal pain, bloating, chills or fever. The chest pain occurred repeatedly 
since the initial onset, and the patient visited a local hospital three months prior to the present study, where he received an electrocardiographic examination indicating myocardial ischemia. The disease was preliminarily diagnosed as coronary heart disease. The rest of the examination and treatment details are unknown.

The week before admission, the patient developed a mild cough and evidently aggravated exertional chest pain that lasted for $>10 \mathrm{~min}$. In order to obtain further diagnosis and treatment, the patient visited Daping Hospital in July 2011, and was admitted to the Department of Cardiovascular Medicine as a patient with coronary heart disease. The patient reported having suffered from a cold 1 week earlier, low mood, loss of appetite and sleep, but had normal stool and urine. The patient had a 10-year history of chronic bronchitis and chronic obstructive emphysema, 15-year history of hypertension and 40-year smoking history (20 cigarettes/day). He had never received systematic blood pressure monitoring or antihypertensive therapy. Physical examination demonstrated a good general state of health with a body temperature of $36.2^{\circ} \mathrm{C}$, a pulse rate of $75 \mathrm{bpm}$, a respiratory rate of $18 \mathrm{bpm}$, and blood pressure of 124/72 $\mathrm{mmHg}$, with normal head and face features. Jugular vein distention was not detected and the thyroid gland was not enlarged. Weakened breathing movement of both lungs, widened intercostal space, reduced tactile fremitus and hyperresonant percussion note were observed. The breath sounds of the two lungs were diminished, but no dry or moist rales were detected. Heart auscultation revealed regular rhythm, normal heart sound without murmur and a heart rate of $75 \mathrm{bpm}$. However, narrowed cardiac dullness was found. Liver and spleen were normal, and no swelling was observed in the lower extremities.

Following hospitalization, electrocardiography (ECG) results indicated a sinus rhythm with a heart rate of $75 \mathrm{bpm}$ and a slightly-depressed ST segment on lead V2-6 of ECG with inverted T wave. Echocardiography demonstrated minor effusion in the heart sac, some reflux across aortic valves, and reduction in the left ventricular diastolic function. Chest X-ray examination revealed increased texture and light transmittance in both lungs, dense nodule shadows in bilateral upper lungs, a long and narrow heart shadow, as well as tortuous and widened aortic arch with arc calcification at its edge (Fig. 1). The bilateral diaphragm was at a lower level than the chest, the intercostal space was widened, and the costophrenic angle was sharp. All these observations suggested chronic bronchitis and chronic obstructive emphysema (Fig. 1). No other abnormalities were present in other examinations, including routine blood, urine and fecal samples, liver and kidney function, blood electrolyte levels, blood glucose, blood lipids, coagulogram, myocardial damage markers, thyroid gland function and abdominal B-mode ultrasound. The patient was preliminarily diagnosed with coronary heart disease, unstable angina and cardiac function of grade 2 (NYHA II) (10), hypertension of grade 3 (11), chronic non-obstructive bronchitis (acute phase) and chronic obstructive emphysema. The patient was administered low-molecular-weight heparin (enoxaparin sodium, $0.4 \mathrm{ml}$ i.p., once/12 h), rosuvastatin calcium tablets $(10 \mathrm{mg}$, once/day), clopidogrel hydrogen sulfate ( $75 \mathrm{mg}$, once/day), aspirin (100 mg, once/day), intravenous infusion with nitroglycerin, and other relevant therapies.
Due to persistent and frequent chest tightness, coronary angiography through the right radial artery was performed on the day of hospitalization after the patient was administered $300 \mathrm{mg}$ clopidogrel hydrogen sulfate and $300 \mathrm{mg}$ aspirin. Angiography revealed a dominant right coronary artery, intimal flap at the left main coronary artery, 30-80\% stenosis of the left main coronary artery opening to the middle anterior descending branch, and nearly total occlusion of the distal segment of the anterior descending branch and the third diagonal branch. In addition, $\sim 90 \%$ proximal circumflex artery stenosis was observed with a TIMI grade 3 , as well as $\sim 30 \%$ right coronary opening stenosis and $\sim 80 \%$ distal right coronary stenosis. Following a balloon angioplasty, the coronary artery stenosis was eliminated following the implantation of two Maverick stents in the anterior descending branch, one in the circumflex artery and another in the right coronary artery. During surgery, the patient experienced two episodes of severe cough, without any other discomfort. Percutaneous coronary intervention (PCI) was conducted within $\sim 2 \mathrm{~h}$, and then the patient was returned to the critical care unit. Following surgery, the aforementioned medications were continued.

At $5 \mathrm{~h}$ after the surgery, the patient suddenly presented symptoms of palpitations, shortness of breath, dizziness, sweating, clammy and pale skin, tachypnea, narrowed pulse beating at a faster speed, reduced blood pressure (65-90/35-58 $\mathrm{mmHg}$ ), left shift of the trachea, decreased right chest breathing mobility, further widened intercostal space, tactile fremitus disappearance, percussive flatness, and disappearance of lower right lung breath sounds during auscultation. The symptoms of the left chest were unchanged. The suspected diagnosis was massive hemothorax in the right thoracic cavity and hemorrhagic shock. Thus, anticoagulant and antiplatelet therapies were immediately terminated, and the patient was subjected to blood transfusion and was administered dopamine to increase the blood pressure. Blood examination suggested that hemoglobin level was decreased from the preoperative level of $150 \mathrm{~g} / \mathrm{l}$ to a postoperative level of $65 \mathrm{~g} / 1$ (normal range 115-156 g/l). A chest X-ray examination suggested that the right thoracic cavity of the patient had a large number of effusions and the mediastinum was broader compared with that on admission (Fig. 2). Ultrasound examination indicated low effusion in the heart sac (as observed earlier), and high effusion and blood clotting (verified as bright red and non-condensing blood through puncture) in the right thoracic cavity. The reason of hemothorax was suspected to be one of the following: i) Vascular injury in the right subclavian artery and brachiocephalic artery resulting in openings to the thoracic cavity and bleeding into the right thoracic cavity; ii) bleeding through the perforated coronary artery into the pericardial cavity, the impaired epicardium and finally the right thorax; iii) aortic dissection leading to bleeding into the right thoracic cavity. Due to the continuous decrease in blood pressure, the loss of consciousness and 70-80\% oxygen saturation with mask inhalation of oxygen, norepinephrine ( $1 \mu \mathrm{g} / \mathrm{kg} / \mathrm{min}$ ) was administered to maintain the blood pressure (approximately $80 / 40 \mathrm{mmHg}$ ) and artificial assisted respiration with tracheal intubation was applied. Simultaneously, an emergency diagnosis by angiography of the coronary artery, aorta, right subclavian artery and truncus brachiocephalicus was conducted once again. Following careful 


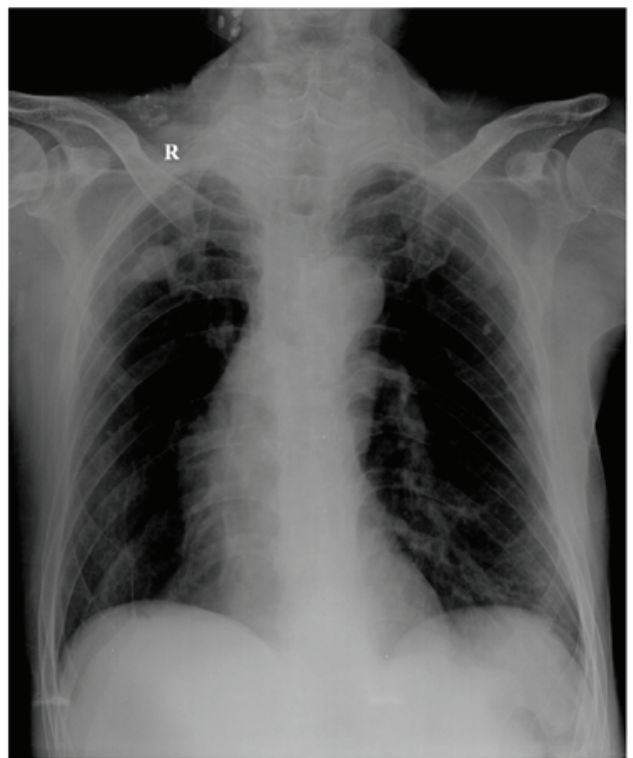

Figure 1. Chest X-ray scan on admission. Increased markings and transmittance were observed in the lungs. Dense nodule shadows was detected in bilateral upper lungs, while the bilateral diaphragmatic muscle was low and flat. The intercostal space was increased, the costophrenic angle was sharp, and the heart shadow was long and narrow. Finally, the aortic arch was tortuous and widened, with arc calcification at its edge. R, right.

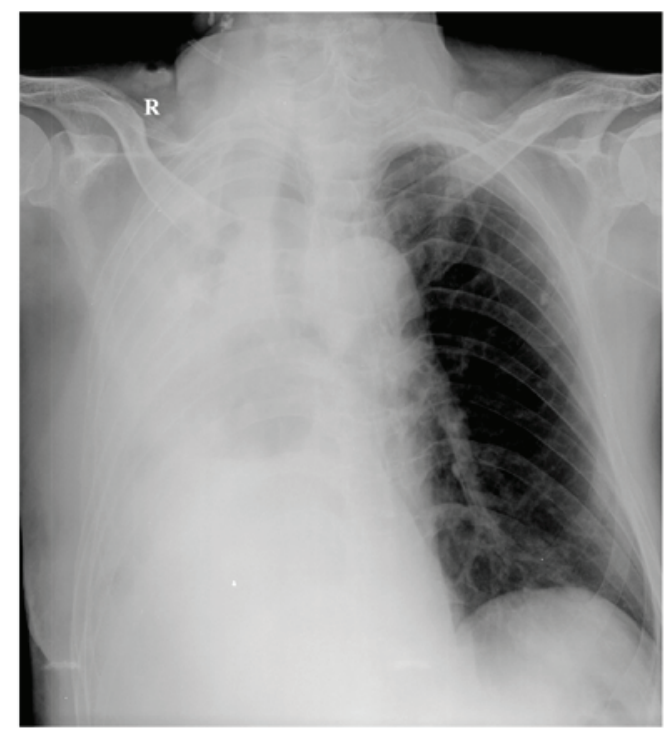

Figure 2. Bedside chest X-ray scan conducted at $5 \mathrm{~h}$ after surgery. The right thoracic cavity presented increased effusion, and the mediastinum was broader compared with that observed on admission.

examination, no artery injury, puncture or aorta dissection was observed. Considering his chronic obstructive pulmonary disease history and intense coughs during the surgery, it was suspected that the hemothorax was caused by rupture of the pleural blood vessels. Therefore, the patient was urgently subjected to right chest examination with a video-assisted thoracoscope after thoracic investigation. Approximately $3,000 \mathrm{ml}$ blood was observed in the right thoracic cavity, where a number of blood clots existed, and the right lung was found to be compressed. After removal of the blood and clots, tearing of the pleural adhesions, with band sizes of

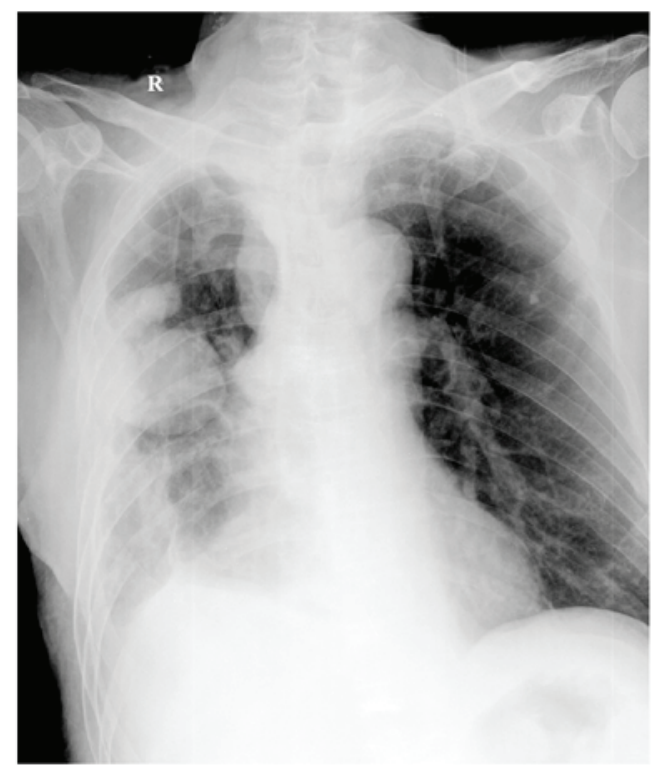

Figure 3. Chest X-ray scan after treatments including thoracic surgery and blood transfusion. The right chest area presented encapsulated effusion and pleural adhesion. $\mathrm{R}$, right.

2.1x2.0, 3.5x 2.9 and $1.8 \times 2.5 \mathrm{~cm}$ was observed at the top of the right chest, right upper chest and right upper mediastinum, respectively. Hemorrhage was also evident in certain areas. Thus, pleural adhesion band solidification and suture hemostasis were performed by an open chest surgery and adhesion band lysis.

Subsequent to treatments including intravenous dopamine/ dobutamine administration and continuous blood transfusion, increase of blood pressure, prevention of infection, cough relief and closed thoracic drainage, the patient recovered to a normal state 2-3 days following the thoracic surgery, with a blood pressure of 110-130/60-70 $\mathrm{mmHg}$ and hemoglobin levels of 130-150 g/l. A chest ultrasound revealed encapsulated effusion, while the volume of fluid did not increase after thoracic puncture. However, encapsulated effusion and pleural adhesion were still observed in the right chest in an X-ray scan (Fig. 3). At 5 days after surgery, the patient was treated with low-molecular-weight heparin (enoxaparin sodium, $0.4 \mathrm{ml}$, subcutaneous injection, once/12 h), clopidogrel hydrogen sulfate (75 mg once/day) and aspirin (100 mg once/day). No further bleeding in relation to the disease was developed. The patient was discharged from the hospital on day 12 after the surgery. During the nearly 1 year of follow-up, the patient was maintained in a normal condition, without severe discomfort, and no hemothorax was detected by ultrasound. Pleural adhesion was detected but no encapsulated effusion was observed.

\section{Discussion}

PCI is an effective therapy for treating coronary artery disease, and hemothorax during PCI is rare (12). The possible causes of hemothorax are vascular injuries along the catheter passage (subclavian artery, brachiocephalic artery, and connected to the thoracic cavity), coronary artery perforation resulting in bleeding into the pericardial 
cavity and then into the thoracic cavity through the injured epicardium, or spontaneous hemorrhage induced by poor blood coagulation (6). By comprehensively analyzing the conditions, spontaneous hemorrhage resulting from poor blood coagulation was excluded in the present case. In addition, the injury of blood vessels (including coronary artery) was also excluded through an angiogram examination. It was eventually verified by an open chest surgery that the complication was caused by multiple-site bleeding at the adhesion bands of the pleura.

Spontaneous and progressive hemothorax is rare in the clinical practice, and is predominantly observed in 20-40-year-old males (13). The occurrence ratio of spontaneous and progressive hemothorax is $\sim 25: 1$ for males:females (7), this may partly ascribe to tobacco smoking among young males that induces the release and formation of inflammatory factors (7). Spontaneous and progressive hemothorax seldom appears in the elderly, and early diagnosis is difficult. Bleeding at the adhesion band of the pleura due to laceration is a cause of spontaneous and progressive hemothorax (2-4). However, the patient in the present study experienced multiple-site bleeding at the adhesion bands of the apex of the right lung, which is very rare. The pleural adhesion band is a localized adhesion occurring between the visceral pleura and the parietal pleura following pleural inflammation (14). The unbalance of the ventilation/blood flow ratio due to the special anatomic structure of the lung apex and easily lead to inflammation in this region and to pleural adhesions when the immune system is weakened (15). It is uncommon for pleural adhesions to be torn or cause hemothorax. Nevertheless, the lung may instantly change from overexpansion to rapid retraction due to abrupt alterations in intrathoracic pressure after coughing, sneezing, breath-holding, deep breathing or sudden changes in body posture. Under such occasions, the adhesion band can be torn or broken by outbursts of tractive force and twisting force, resulting in bleeding. The range of motion of the diaphragm may serve an important role in the onset of this condition.

Pleural pressure is negative due to a suction effect caused by lung recoil. Negative pressure within the pleural cavity may cause bleeding in the pleura, which is difficult to be stopped by normal hemostasis, and thus a large amount and long duration of bleeding may be observed, causing severe bleeding and shock $(14,16)$. Due to quick bleeding, defibrinogenation is not complete and coagulation occurs. Blood clots are frequently formed at the early stages in up to $84 \%$ of patients (2). In the present study, the 76-year-old patient suffered from massive hemorrhage in the chest soon after PCI, thus, pleural hemorrhage was initially suggested to be associated with surgical injury prior to further angiography examination. Eventually, it was observed that pleural bleeding was due to the tear of pleural adhesion bands. A reasonable explanation for the occurrence of pleural bleeding can be provided, considering the patient's chronic obstructive pulmonary disease history and that the angina pectoris of coronary artery disease occurred with cough. More specifically, coughing possibly resulted in the laceration of preexisting adhesion bands, and the bleeding continued at a faster pace due to the lack of vascular smooth muscle and thus contraction of blood vessels in the pleura, as well as due to high blood pressure at the top of the parietal layer of the thoracic cavity as part of the systemic circulation, and the effect of negative pressure in the thoracic cavity. Massive hemothorax was further promoted by the use of large-dose anticoagulant and antiplatelet agents.

The case reported in the present study suggests that, in clinical practice, it should be considered that the bleeding may be caused by laceration of preexisting pleural adhesion bands, following exclusion of other common reasons for hemothorax. Early diagnosis of this condition may be established according to the following features: i) hemothorax occurring suddenly in a healthy young patients; ii) forceful chest movements and strenuous exercise prior to onset; iii) progressive bleeding and shock; iv) physical signs of effusion on one side of the thoracic cavity with corresponding X-ray features; and v) blood sampling through thoracentesis.

For non-progressively aggravated spontaneous hemothorax in which the bleeding is slow and at a small amount, conservative treatments can be applied in patients with light compression of lung tissue, good general condition, unchanged breathing, pulse and blood pressure. These treatments include rest, oxygen inhalation, and prevention of infection, blood volume supplement and application of hemostatic drugs. If necessary, thoracentesis or placement of a thoracic closed drainage tube can be used, which not only helps the reengagement of previously compressed lungs, but also stops the bleeding and provides a method to observe whether progressive bleeding appears in the thoracic cavity $(17,18)$. With a growing number of patients receiving PCI therapy, the rare but potentially fatal complications should receive worthy attention during the perioperative period. Chest contrast-enhanced computed tomography (CT) to examine the leural adhesions may help to improve the evaluation of the risk of hemothorax. The use of antitussive drugs for the patients with heart disease, especially the elderly, prior to PCI may reduce the occurrence of the severe cough and thereby avoiding its caused hemothorax during the therapy. Recently, electrical hemostasis, suture at the bleeding point and removal of blood clots in the thoracic cavity with video-assisted thoracoscopic surgery have been observed to have certain advantages, including reduced trauma, good therapeutic effect, quick recovery and easily accepted by the patient (19). However, special and expensive equipment with demanding skills are required for such treatment, thus it is difficult to perform these procedures at local hospitals. Patients presenting shock or progressively aggravated bleeding, or when no lung expansion after thoracentesis or closed drainage of the thoracic cavity is observed in a patient with or without suspected active bleeding, then surgery should be performed as soon as possible. In fact, the majority of such patients eventually require surgical intervention (20-22).

In conclusion, spontaneous and progressive hemothorax caused by bleeding at the adhesion band of the pleura is a life-threatening complication during the perioperative period of PCI. The current study reported the case of an elderly male patient with coronary heart disease who presented multiple-site bleeding at pleural adhesions following PCI. Patients with a history of lung disease are at a higher risk of hemothorax. Early diagnosis and effective treatment including surgical intervention may significantly improve the prognosis for these patients. 


\section{References}

1. Hentel K, Brill PW and Winchester P: Spontaneous hemopneumothorax. Pediatr Radiol 32: 457-459, 2002.

2. Hsu NY, Hsieh MJ, Liu HP, Kao CL, Chang JP, Lin PJ and Chang CH: Video-assisted thoracoscopic surgery for spontaneous hemopneumothorax. World J Surg 22: 23-27, 1998.

3. Homma T, Sugiyama S, Kotoh K, Doki Y, Tsuda M and Misaki T: Early surgery for treatment of spontaneous hemopneumothorax. Scand J Surg 98: 160-163, 2009.

4. Ohmori K, Ohata M, Narata M, Iida M, Nakaoka Y, Irako M, Kitamura K, Nakamura S, Natori H and Sezaki Y: 28 cases of spontaneous hemopneumothorax. Nihon Kyobu Geka Gakkai Zasshi 36: 1059-1064, 1998 (In Japanese).

5. Tulay CM and Aygün M: Emergency surgery for spontaneous hemopneumothorax. J Coll Physicians Surg Pak 24: 435-447, 2014.

6. Levine GN, Bates ER, Blankenship JC, Bailey SR, Bittl JA, Cercek B, Chambers CE, Ellis SG, Guyton RA, Hollenberg SM, et al: 2011 ACCF/AHA/SCAI Guideline for Percutaneous Coronary Intervention: A report of the American College of Cardiology Foundation/American Heart association task force on practice guidelines and the society for cardiovascular angiography and interventions. J Am Coll Cardiol 58: e44-e122, 2011.

7. Onuki T, Goto $\mathrm{Y}$, Kuramochi M, Inagaki $\mathrm{M}$ and Sato $\mathrm{Y}$ : Spontaneous hemopneumothorax: Epidemiological details and clinical features. Surg Today 44: 2022-2027, 2014.

8. Patel MR, Calhoon JH, Dehmer GJ, Grantham JA, Maddox TM, Maron DJ and Smith PK: ACC/AATS/AHA/ASE/ASNC/ SCAI/SCCT/STS 2017 Appropriate use criteria for coronary revascularization in patients with stable ischemic heart disease: A report of the American College of Cardiology Appropriate use criteria task force, American Association for Thoracic Surgery, American Heart Association, American Society of Echocardiography, American society of nuclear cardiology, society for cardiovascular angiography and interventions, society of cardiovascular computed tomography, and society of thoracic surgeons. J Am Coll Cardiol 69: 2212-2241, 2017.

9. Zheng X, Curtis JP, Hu S, Wang Y, Yang Y, Masoudi FA, Spertus JA, Li X, Li J, Dharmarajan K, et al: Coronary catheterization and percutaneous coronary intervention in China: 10 -year results from the China PEACE-retrospective CathPCI study. JAMA Intern Med 176: 512-521, 2016.

10. St John Sutton M, Ghio S, Plappert T, Tavazzi L, Scelsi L, Daubert C, Abraham WT, Gold MR, Hassager C, Herre JM, et al: Cardiac resynchronization induces major structural and functional reverse remodeling in patients with New York Heart Association class I/II heart failure. Circulation 120: 1858-1865, 2009.
11. Touyz RM and Dominiczak AF: Hypertension guidelines: Is it time to reappraise blood pressure thresholds and targets? Hypertension 67: 688-689, 2016.

12. Robinson NM, Thomas MR and Jewitt DE: Spontaneous haemothorax as a complication of anti-coagulation following coronary angioplasty. Respir Med 89: 629-630, 1995.

13. Azfar Ali H, Lippmann M, Mundathaje U and Khaleeq G Spontaneous hemothorax: A comprehensive review. Chest 134: 1056-1065, 2008

14. Kim ES, Kang JY, Pyo CH, Jeon EY and Lee WB: 12-year experience of spontaneous hemopneumothorax. Ann Thorac Cardiovasc Surg 14: 149-153, 2008.

15. De Smedt A, Vanderlinden E, Demanet C, De Waele M, Goossens A and Noppen M: Characterisation of pleural inflammation occurring after primary spontaneous pneumothorax. Eur Respir J 23: 896-900, 2004

16. Chang YT, Dai ZK, Kao EL, Chuang HY, Cheng YJ, Chou SH and Huang MF: Early video-assisted thoracic surgery for primary spontaneous hemopneumothorax. World J Surg 31: 19-25, 2007.

17. Kakaris S, Athanassiadi K, Vassilikos K and Skottis I: Spontaneous hemopneumothorax: A rare but life-threatening entity. Eur J Cardiothorac Surg 25: 856-858, 2004.

18. Ng CSh and Yim AP: Spontaneous hemopneumothorax. Curr Opin Pulm Med 12: 273-277, 2006.

19. Wu YC, Lu MS, Yeh CH, Liu YH, Hsieh MJ, Lu HI and Liu HP: Justifying video-assisted thoracic surgery for spontaneous hemopneumothorax. Chest 122: 1844-1847, 2002.

20. Inafuku K, Maehara T, Yamamoto T and Masuda M: Assessment of spontaneous hemopneumothorax: Indications for surgery. Asian Cardiovasc Thorac Ann 23: 435-438, 2015.

21. Haciibrahimoglu G, Cansever L, Kocaturk CI, Aydogmus U and Bedirhan MA: Spontaneous hemopneumothorax: Is conservative treatment enough? Thorac Cardiovasc Surg 53: 240-242, 2005.

22. de Perrot M, Deléaval J, Robert J and Spiliopoulos A: Spontaneous hemopneumothorax-results of conservative treatment. Swiss Surg 6: 62-4, 2000.

This work is licensed under a Creative Commons Attribution-NonCommercial-NoDerivatives 4.0 International (CC BY-NC-ND 4.0) License. 